Artículo Original

\title{
Relación entre tabaquismo y las principales enfermedades no transmisibles en El Salvador
}

\author{
DOI 10.5377/alerta.v5i1.11753 \\ Liliam Herrera de Hurtado \\ Fondo Solidario para la Salud, San Salvador, El Salvador \\ ${ }^{*}$ Correspondencia \\ $\square$ liliamherrera@yahoo.com \\ (1) $0000-0002-7998-9311$
}

\section{ACCESO ABIERTO}

Relationship between smoking and the most relevant noncommunicable diseases in El Salvador

\section{Citación recomendada:} Herrera de Hurtado L. Relación entre el tabaquismo y las principales enfermedades no transmisibles en El Salvador, 2019. Alerta. 2021:5(1):26-32 DOI: $10.5377 /$ alerta.v5i1.11753

Recibido:

24 de junio de 2021

\section{Aceptado:}

20 de diciembre de 2021

\section{Publicado:}

27 de enero de 2022

\section{Contribución de autoría:} LHH: Contribución total de la investigación.

\section{Conflicto de intereses:}

La autora declara no tener conflictos de interés en el estudio.

\begin{abstract}
Resumen
Introducción. El consumo de tabaco es uno de los principales factores de riesgo para el desarrollo de enfermedades no transmisibles, como la hipertensión, la diabetes mellitus, el cáncer, las enfermedades cardiovasculares y la insuficiencia renal crónica. Objetivo. Analizar la relación entre el tabaquismo y los diagnósticos de hipertensión, diabetes, insuficiencia renal y cáncer en usuarios de la red de salud de El Salvador, 2019. Metodología. Se realizó un estudio transversal analítico con una población de 63061 usuarios con antecedente de tabaquismo y con diagnóstico de hipertensión, diabetes mellitus, insuficiencia renal y/o cáncer. Resultados. La prevalencia de tabaquismo a nivel de país fue de 1,2\%, con predominio de tabaquismo pasivo, seguido de tabaquismo activo y exfumadores. En las mujeres predomina el tabaquismo indirecto, con una prevalencia de $0,7 \%$, y en los hombres el consumo directo de tabaco, con una prevalencia de $0,1 \%$; el tabaquismo pasivo predomina en las personas entre los 25 a 59 años (48,1 \%). Finalmente, se encontró una correlación positiva de la prevalencia de consumo de tabaco con el diagnóstico de hipertensión arterial $(0,4)$, así como de cáncer $(0,4)$, seguido de diabetes mellitus $(0,2)$ e insuficiencia renal crónica $(0,09)$. Conclusiones. El tabaquismo está relacionado con el diagnóstico de hipertensión, diabetes, insuficiencia renal y cáncer en usuarios de la red de salud de El Salvador.

Palabras clave

Tabaquista, no fumadores, diabetes mellitus, insuficiencia renal.
\end{abstract}

\section{Abstract}

Introduction. Tobacco use is one of the main risk factors for the development of non-communicable diseases, such as hypertension, diabetes mellitus, cancer, cardiovascular diseases and chronic kidney failure. Objective. To analyze the relationship between smoking and the diagnoses of hypertension, diabetes, kidney failure and cancer in users of the $\mathrm{EI}$ Salvador health network, 2019. Methodology. An analytical cross-sectional study was carried out with a population of 63,061 users with a history of smoking and a diagnosis of hypertension, diabetes mellitus, kidney failure and / or cancer. Results. The prevalence of smoking at the country level was (1,2\%), with passive smoking predominating, followed by active smoking and exsmokers. Indirect smoking predominates in women, with a prevalence of $(0,7 \%)$, and in men, direct tobacco consumption predominates with a prevalence of $(0,1 \%)$, passive smoking predominates in people among the 25 to 59 years $(48,1 \%)$. Finally, a positive correlation was found between the prevalence of tobacco consumption with the diagnosis of arterial hypertension $(0,4)$, as well as that of cancer $(0,4)$, followed by diabetes mellitus $(0,2)$ and insufficient chronic kidney $(0,09)$. Conclusions. Smoking is related to the diagnosis of hypertension, diabetes, kidney failure and cancer in users of the El Salvador health network.

Keywords

Smokers, non-smokers, diabetes mellitus, renal insufficiency.

\section{Introducción}

El tabaquismo es considerado un factor desencadenante de las principales enfermedades no transmisibles (ENT), como enfermedades cardiovasculares, enfermedad pulmonar obstructiva crónica, cáncer de pulmón, diabetes mellitus, insuficiencia renal, entre otras; así también, el tabaquismo se considera la principal causa de muerte que se puede prevenir a nivel mundial'. Según datos de la Organización Mundial de la Salud (OMS), el tabaquismo, ya sea activo o de segunda mano, o conocido también como tabaquismo pasivo, causa la muerte a más de 8 millones de personas anualmente; de esta cantidad, más de 7 millones corresponden a tabaquistas activos y el resto (1,2 millones) son no fumadores expuestos al humo de segunda mano. En general, de 1,3 millones de consumidores de tabaco, el $80 \%$, viven en países pobres o con bajos ingresos ${ }^{2}$. 
Las ENT se encuentran entre las primeras causas de muerte ocurridas en el mundo?. Estas enfermedades representan más del $75 \%$ de las muertes en un año, y aportan las primeras causas de muerte prematura o discapacidad en las personas que las están padeciendo ${ }^{3}$.

En El Salvador, el consumo de tabaco es cada vez más evidente en la población adolescente y adulta joven. Este grupo de personas son consideradas de mayor vulnerabilidad para el inicio temprano de consumo de tabaco, así como para adquirir el riesgo de desarrollar enfermedades crónicas no transmisibles. Según la Encuesta Mundial sobre Tabaco en Jóvenes (GYTS por sus siglas en inglés) realizada en El Salvador en el 2009, el $14,6 \%$ de los estudiantes de 13 a 15 años consume algún producto de tabaco; de ellos, el $18,2 \%$ son hombres y el $11 \%$ mujeres. Para el 2015, estos datos aumentaron a un 32,4\% en los estudiantes de las mismas edades 4 .

En un estudio llevado a cabo en El Salvador se mencionan las principales ENT con antecedente de tabaquismo en el 2016, entre las que se encontraron la enfermedad isquémica del corazón, la enfermedad pulmonar obstructiva crónica, infecciones respiratorias, cáncer de pulmón, diabetes mellitus (DM), enfermedad cardiovascular hemorrágica, enfermedad hipertensiva y otras enfermedades cardiovasculares ${ }^{5}$. En este sentido, existe evidencia de una posible relación del tabaquismo con principales diagnósticos de ENT en el país; sin embargo, es necesario actualizar la información científica y estimar esta relación, sobre todo en grupos de personas que asisten a consulta en los principales centros de salud del primer nivel de atención en el país.

Por lo anterior, el estudio buscó analizar la prevalencia de tabaquismo activo, pasivo y exfumador, así como la relación de estas con los diagnósticos de hipertensión arterial $(H T A)$, diabetes mellitus, insuficiencia renal y cáncer en usuarios de la red de salud de El Salvador durante el 2019, de tal manera que los resultados incidan en las políticas de control de tabaco como la cesación, reducción de consumo y ambientes libres de humo y en acciones tempranas y concretas para la prevención y control de tabaco ${ }^{6}$. Estas estrategias de intervención son clave para reducir enfermedades y muertes ocasionadas por su consumo y para la reducción de costos sanitarios, los cuales afectan las economías de distintos países $5^{6,7,8}$.

\section{Metodología}

Estudio transversal analítico,10. Los datos recolectados permitieron medir la prevalencia de tabaquismo en usuarios de la red de salud pública, así como estimar la proporción de diagnósticos de las ENT con el tabaquismo en El Salvador.

La unidad de análisis fueron los registros administrativos de información de usuarios de la red de salud pública en el Sistema de Morbilidad y Mortalidad en línea de El Salvador (SIMMOW) ${ }^{11}$.

Para el estudio se tomó a toda la población que consultó en el 2019, con un total de 5 169912, de los cuales 63061 participantes cumplieron los siguientes criterios: usuario registrado en el SIMMOW durante el 201911,12, con el antecedente de tabaquista activo, tabaquista pasivo o exfumador, además de tener cualquiera de los diagnósticos siguientes: HTA, DM, insuficiencia renal y cáncer, así como tener cualquier edad, sexo y procedencia.

La técnica de recolección de datos utilizada fue la revisión de los registros de SIMMOW. En este sentido, la base de datos incluye información de usuarios que consultan en los diferentes centros de atención pública, ya sea del primero, del segundo o del tercer nivel.

Para la recolección de datos se estructuró una ficha que cuenta con 8 preguntas cerradas y categorizadas como variables de tipo nominal, entre ellas: sexo, procedencia urbano o rural, diagnóstico de HTA, DM, insuficiencia renal, cáncer, antecedente de tabaquismo activo, tabaquismo pasivo y exfumador. La única variable de tipo escala fue la edad de los usuarios. No se validó el instrumento de recolección de datos.

Por otro lado, se efectuó el análisis de datos descriptivos, por medio del cual se obtuvieron proporciones de tabaquismo activo, pasivo y exfumador, clasificados por sexo, rangos de edad y área geográfica. Los resultados del cálculo de prevalencia de tabaquismo activo, pasivo, y exfumador se expresan en porcentajes a escala nacional.

La estimación de la correlación de variable de tabaquismo y los principales diagnósticos de ENT se hizo con el programa Excel versión 2010. La prueba estadística utilizada para evaluar la normalidad de los datos fue el test de Kolmogorov-Smirnov; el valor del estadístico proporcionado por dicho test fue de 0,705 , con un $p$-valor de $p=0,702$, lo cual indica una distribución normal. Para el cálculo de la correlación de variables se utilizó prueba estadística de coeficiente de correlación de Pearson ${ }^{13}$.

Los resultados se presentan y analizan en gráficas de barras y tablas, utilizando Excel versión 2010.

En la investigación se respetaron los principios éticos. El anteproyecto de esta 
investigación fue revisado y aprobado sin restricciones por el Comité de Ética de la Universidad Evangélica de El Salvador.

\section{Resultados}

De 63061 usuarios de la red de salud pública con antecedente de tabaquismo registrados en la base de datos del SIMMOW 2019, predominó el sexo femenino (63\%). Al agrupar a la población usuaria con antecedente de tabaquismo por edad, el grupo etario que predomina es el que se encuentra entre 25 a 59 años (39,2\%), seguido por el grupo $\geq 60$ años (19,9\%). Por otro lado, el rango de 15 a 24 años representa un 17,6\% de tabaquismo en general, los menores de 5 años representan el $14,5 \%$ de tabaquismo pasivo y el rango de 5 a 14 años, el 8,8\% en las diferentes variedades de tabaquismo.

En cuanto a las ENT con antecedente de tabaquismo, un $69 \%$ de los usuarios presenta diagnóstico de HTA más tabaquismo, le siguen el diagnóstico de DM más tabaquismo, con un $27,5 \%$, y el diagnóstico de insuficiencia renal y tabaquismo, con un 3,3\%. Por otro lado, el diagnóstico de cáncer, inde- pendientemente del tipo, con el antecedente de tabaquismo, corresponde a un 0,1\% (Figura 1).

Los usuarios con principales diagnósticos de ENT presentan una prevalencia de tabaquismo de 1,2\%.

En la Figura 2 se observa que predomina la condición de tabaquismo pasivo, seguido del tabaquismo activo, y en menor prevalencia los exfumadores.

La prevalencia de tabaquismo pasivo es de $0,7 \%$ en el sexo femenino y del 0,2\% en el sexo masculino. En la prevalencia del tabaquismo activo y de exfumador predomina el sexo masculino, con 0,11\%, y 0,08 \% en el sexo femenino (Figura 3).

La prevalencia de tabaquismo por rangos de edad es mayor en las personas de 25 a 59 años (0,4\%), seguida las personas de 60 años o más (0,2\%); sin embargo, se observa la presencia de tabaquismo en menores de 19 años, considerando que en los de 0 a 9 años este tipo de tabaquismo corresponde exclusivamente al tabaquismo pasivo o de segunda mano en un 0,2\%; por otro lado, en las personas de 10 a 24 años la prevalencia de tabaquismo es de $0,1 \%$. (Figura 4)

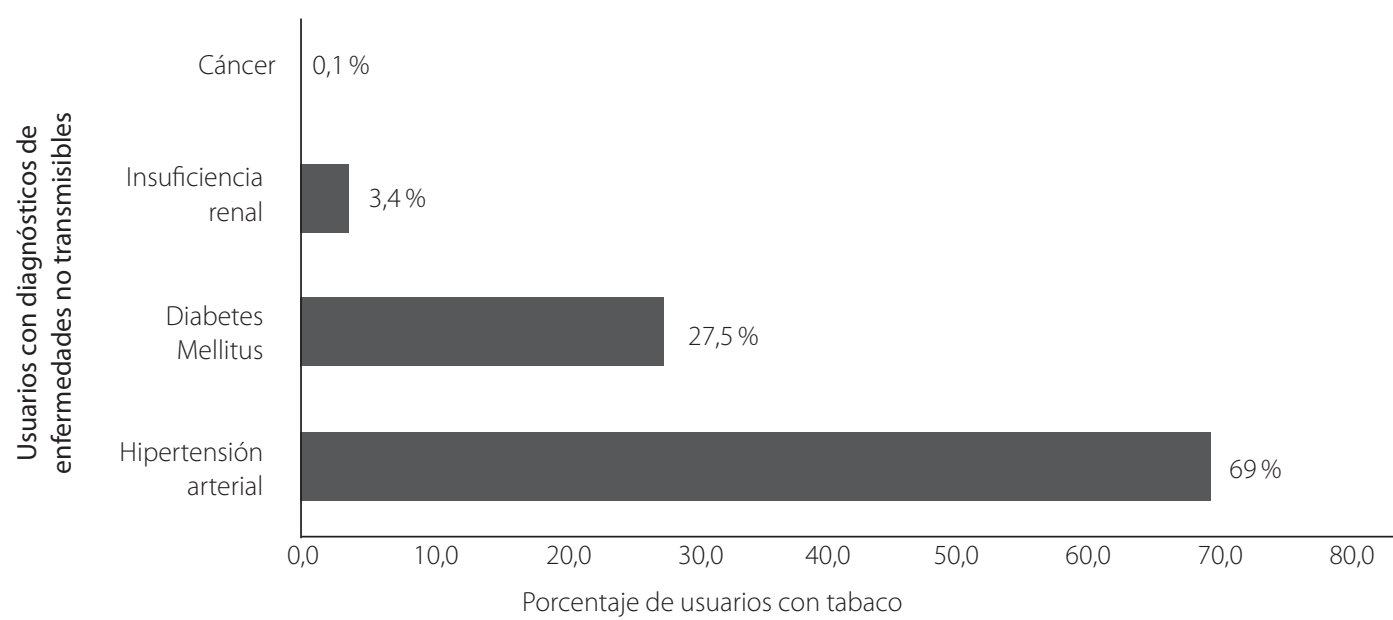

Figura1. Distribución de usuarios de la red de salud con diagnóstico de enfermedades no transmisibles y antecedente de tabaquismo, período 2019.

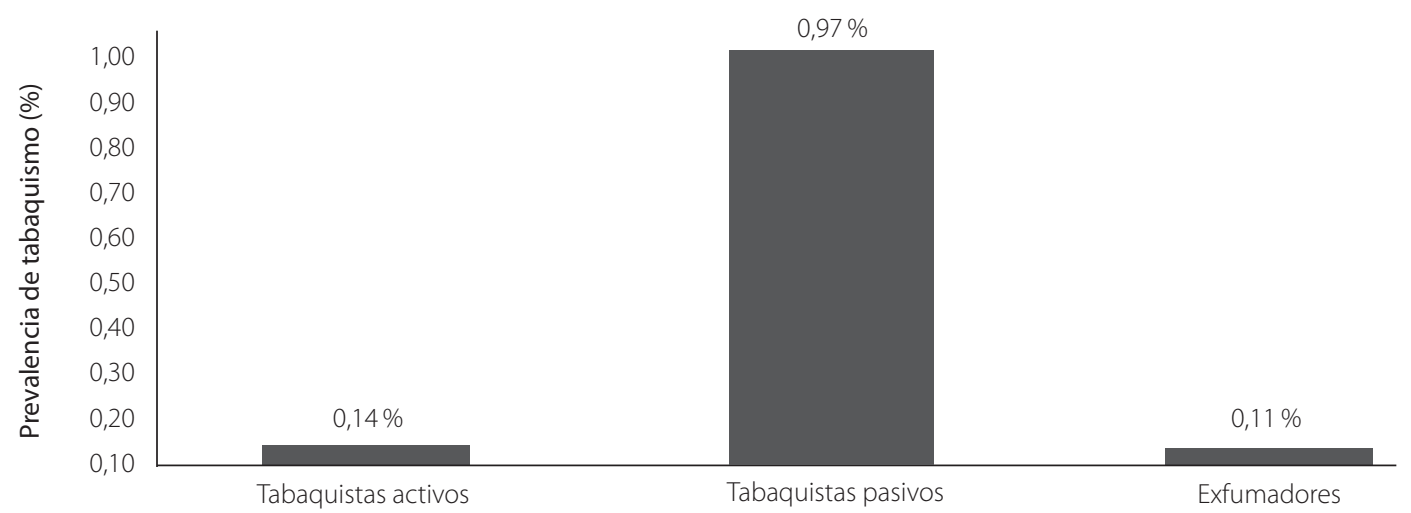

Figura 2. Prevalencia de tabaquismo en usuarios de la red de salud, período 2019. 
La Tabla 1 muestra los resultados de medición de correlación estimados. Para el diagnóstico de HTA con el tabaquismo, el índice de correlación lineal de Pearson fue de 0,44 (moderada). Para el diagnóstico de DM y tabaquismo, el índice de correlación lineal de Pearson es de 0,21 (débil). Por otro lado, la prevalencia de tabaquismo y la proporción de usuarios con diagnóstico de insuficiencia renal presenta un valor de correlación de 0,09 (inexistente). También se calculó la correlación de la prevalencia de tabaquismo y la proporción de usuarios con diagnóstico de cáncer, independientemente del tipo, y el resultado de correlación lineal de Pearson fue de 0,39 (moderada).

\section{Discusión}

Se analizó la correlación del tabaquismo con principales ENT en usuarios de la red de salud del período 2019 y la prevalencia de tabaquismo desagregada por sexo y rangos de edad.

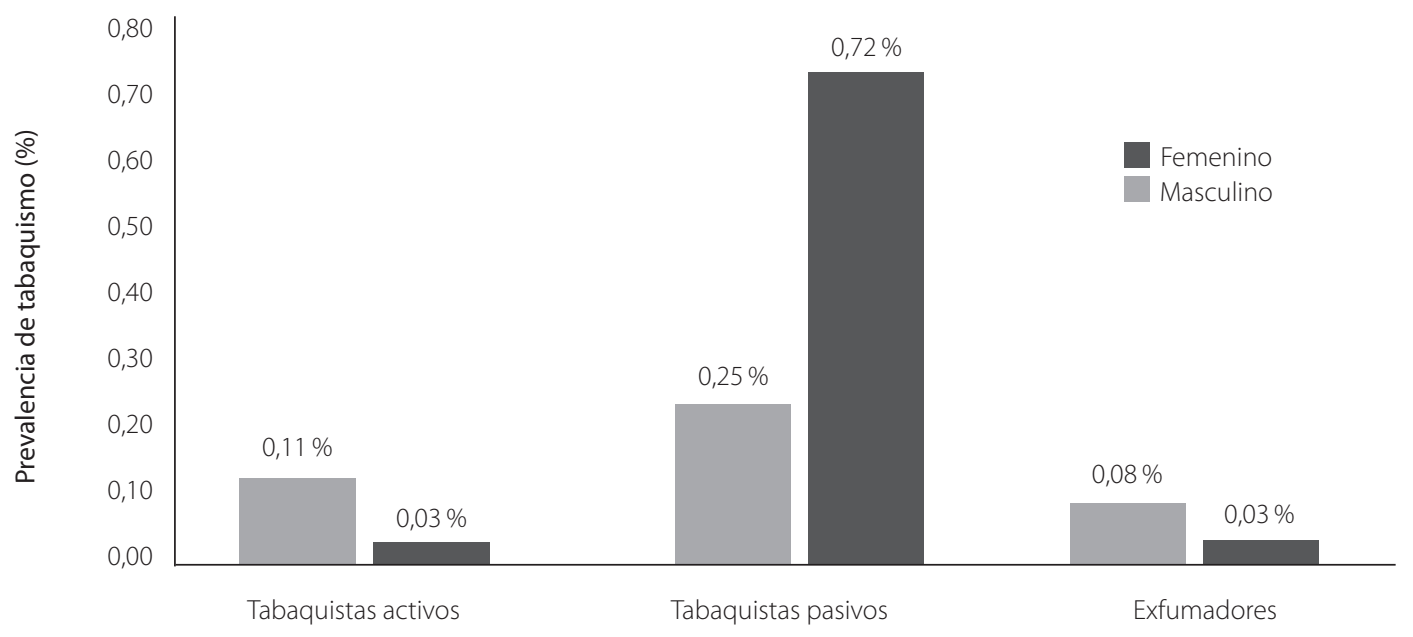

Figura 3. Prevalencia de tabaquismo en usuarios de la red de salud desagregada por sexo y condición tabáquica, período 2019.

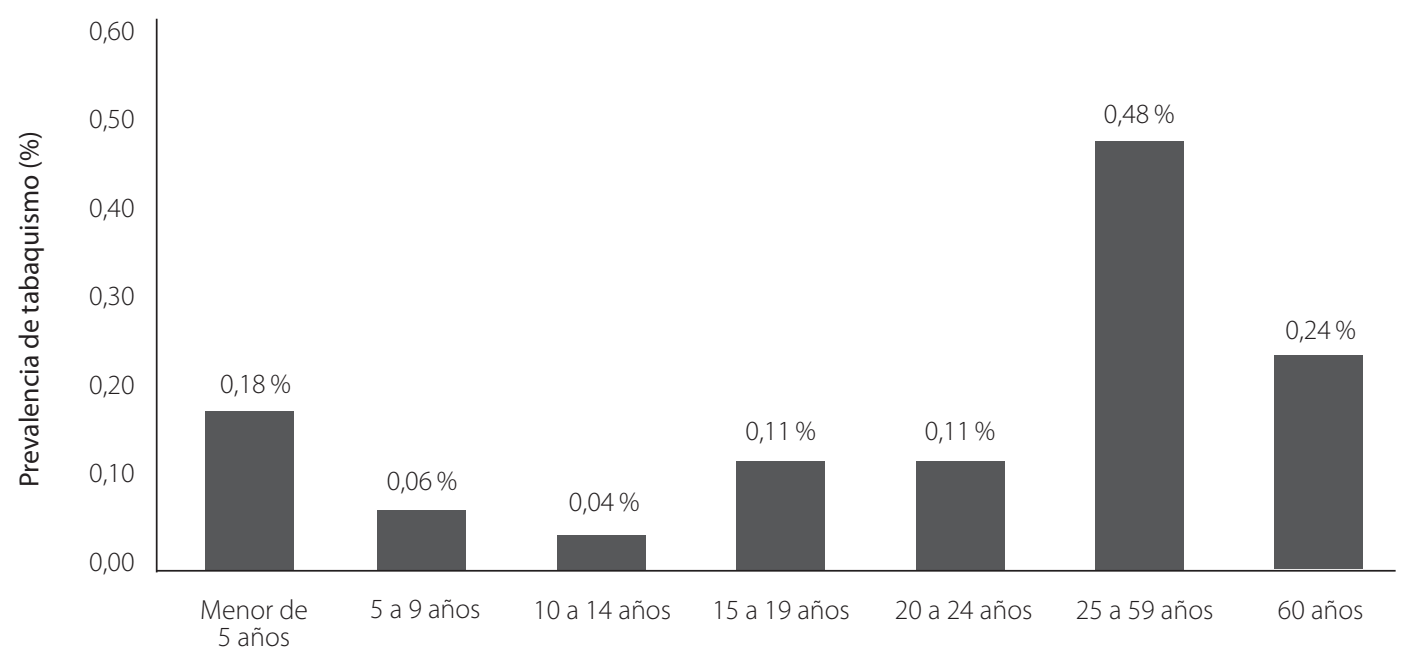

Figura 4. Prevalencia de Tabaquismo por rangos de edad en usuarios de la red de salud, 2019.

Tabla 1. Correlación de la prevalencia de tabaquismo y enfermedades no transmisibles en usuarios de la red de salud, período 2019.

\begin{tabular}{lll}
\hline Diagnóstico de ENT & Correlación de Pearson rho & Significancia bilateral $p$ \\
Hipertensión arterial & 0,44 (moderada) & 0,15 \\
Diabetes Mellitus & 0,21 (débil) & 0,50 \\
Insuficiencia renal & 0,09 (inexistente) & 0,77 \\
Cáncer & 0,39 (moderada) & 0,20 \\
\hline
\end{tabular}


En los resultados de la correlación de ENT y tabaco se evidencia un índice de correlación lineal positiva de tabaquismo (ya sean fumadores activos, pasivo o exfumadores) con las enfermedades no transmisibles estudiadas.

Según la Encuesta Nacional de Adicciones (ENA 2008) realizada en México, el tabaquismo es un factor relacionado a ENT, principalmente enfermedades cardiovasculares, cáncer, enfermedades respiratorias crónicas y $\mathrm{DM}^{14}$.

Otro estudio efectuado en El Salvador en el 2016 evidencia el antecedente de tabaquismo en personas con ENT y muestra que en ese año fallecieron personas diagnosticadas con ENT que tenían antecedente de tabaquismo, entre ellas: enfermedad isquémica del corazón (524), enfermedad pulmonar obstructiva crónica (296), infecciones del tracto respiratorio inferior (258), cáncer de tráquea, bronquios y pulmón (155), DM (132), insuficiencia renal y otros diagnósti$\cos (151)^{5}$.

En un estudio sobre la relación entre tabaquismo y enfermedades cardiovasculares se muestra que la combinación de partículas de nicotina y alquitrán deviene en desarrollo de cardiopatías y aumento de la presión arterial15,16.

Los tabaquistas en general tienen $30 \%$ a $40 \%$ de probabilidad de desarrollar DM que aquellos que no lo son, de tal manera que mientras más cigarrillos fume o que exista mayor exposición al humo de tabaco, más alto es su riesgo de desarrollar $\mathrm{DM}^{1,17}$.

La prevalencia de tabaquismo en usuarios de la red de salud encontrada en este estudio es baja en comparación con los datos que se presentan en la Encuesta Nacional de Alcohol y Tabaco realizada en El Salvador en el 2014, en la que se evidencia que la prevalencia de tabaquismo en El Salvador fue de $35,1 \%{ }^{18}$.

El tabaquismo pasivo es un problema que afecta sobre todo a niños y mujeres, lo que los convierte en grupos de riesgo para desarrollar enfermedades relacionadas a esta condición. Esto duplica la incidencia de enfermedades respiratorias como neumonía, bronquitis y bronquiolitis y asma ${ }^{19}$.

En la Encuesta Mundial de Tabaquismo en Jóvenes (GYTS por sus siglas en ingles), efectuada en El Salvador en el 2015, se identificó que los adolescentes y los escolares se encuentran altamente expuestos al tabaco de segunda mano en lugares públicos, cerrados o abiertos 4 .

El tabaquismo activo y pasivo es mayor en las personas de 25 a 59 años. Un estudio llevado a cabo en Cuba muestra que la prevalencia de tabaquismo activo se encuen- tra principalmente en el rango de 40 a 49 años ${ }^{20}$.

Respecto al tabaquismo activo, los resultados de la investigación muestran que empieza en el rango de 10 a 14 años de edad y que incrementa entre 15 a 19 años. En la Encuesta Mundial de Tabaco en Jóvenes se evidencia que las personas se inician en el consumo de tabaco antes de cumplir los 15 años 21,22 .

Las estadísticas de diversos países revelan un incremento de consumo de tabaco en los últimos años en adolescentes entre los 12 y 14 años edad. Aproximadamente 25 millones de niños y 13 millones de niñas de 13 a 15 años en la región de las Américas fuman cigarrillos o consumen tabaco sin humo ${ }^{23}$.

Las mujeres tienen una prevalencia de tabaquismo mayor que la de los hombres debido a que estas constituyen la población con mayor exposición al humo de segunda mano; en este sentido, el tabaquismo pasivo es el que predomina en las mujeres. Por otro lado, el consumo activo de tabaco es mayor en hombres, evidenciando la continuación de esta tendencia. Según el Atlas del Tabaco de la OMS, el consumo activo de tabaco a nivel global es mayor en hombres que en mujeres de 15 años o más ${ }^{23}$. Otros estudios mencionan que la prevalencia de consumo de tabaco es mayor en hombres que en mujeres, manteniendo la tendencia del inicio de tabaquismo activo en la población adolescente respecto al año 2016-2018 15,24,25.

Una de las limitantes de esta investigación fue el registro del antecedente de tabaquismo en la base de SIMMOW. Es de considerar que el registro de esta característica de antecedente de tabaquismo se inició en el período 2019 a través del dato de los censos de consulta registrados, por lo que es posible que no se haya incluido en el total de la población de usuarios que consultó con este antecedente en este período. Esta situación lleva a un registro menor de casos de pacientes con ENT y el antecedente de tabaquismo, por lo tanto, los resultados presentados en este estudio deben tomarse con cautela, ya que la actualización de los datos en un nuevo estudio puede presentar resultados diferentes.

También debe tenerse en cuenta que el $98 \%$ de los usuarios del estudio provienen del primer nivel de atención y el resto de los servicios de atención del segundo y del tercer nivel; es decir, que la mayoría de la población que consulta y está registrada en el SIMMOW del 2019 proviene de los centros del primer nivel de atención en salud del país. 
El tabaquismo pasivo está presente en todas las edades, desde los menores de 5 años y en mayor proporción en las personas de 25 a 59 años. Lo anterior muestra la necesidad de incidir en acciones dirigidas al cumplimiento de la Ley para el Control de Tabaco $^{13}$, por lo que se recomienda verificar las intervenciones realizadas y fortalecer el apoyo multisectorial con diversas instituciones públicas y privadas que trabajan actualmente en la problemática.

\section{Conclusiones}

Los resultados de la correlación estadística de Pearson con la prevalencia de tabaquismo y proporción de usuarios con el diagnóstico de principales ENT fueron positivas. Existe una mayor correlación entre el consumo de tabaco y la HTA, seguida del cáncer, la DM y finalmente la insuficiencia renal.

La prevalencia de tabaquismo pasivo predomina en el sexo femenino, distribuida en todas las edades, con inicio en menores de 5 años, predominando en mayor proporción en el rango de 25 a 59 años.

La prevalencia del tabaquismo activo se presenta en mayor proporción en el sexo masculino, con predominio en el rango de 25 a 59 años. Así también, el tabaquismo activo se encuentra en el rango de 10 a 14 años, incrementando la prevalencia en el rango de 15 a 19 años.

\section{Agradecimiento}

A la dirección del FOSALUD, por la autorización del uso de la base de datos de SIMMOW 2019 para realizar el estudio, y al Comité de Ética de la Universidad Evangélica de El Salvador, por aprobar el estudio.

\section{Financiamiento}

Se realizó con fondos propios de la investigadora.

\section{Referencias bibliográficas}

1. Casanova C, Trasancos M. Tabaco y diabetes como factores de riesgo de enfermedades no transmisibles. Rev. Electrónica Dr. Zoilo E. 2014;39(9): e1029-3027. Disponible en: http://revzoilomarinello.sld.cu/index.php/ zmv/article/view/154/275

2. Organización Mundial de la Salud (OMS). Tabaco, datos y cifras. 2019. Fecha de consulta: 30 de junio de 2021. Disponible: https://www.who.int/es/news-room/factsheets/detail/tobacco
3. Varona P, Herrera D, García R, Bonet M, Romero T, et al. Mortalidad atribuible al tabaquismo en Cuba. Rev. Cubana de Salud Pública. 2009;35(2):13. Disponible en: https://www.scielosp.org/pdf/ rcsp/2009.v35n2/10.1590/S086434662009000200015/es

4. Fondo Solidario para la Salud (Fosalud). Informe de país de la Encuesta Mundial sobre Tabaco en Jóvenes, GYTS, El Salvador 2015. San Salvador, El Salvador. Organización Mundial de la Salud (OMS). 2016. 20 p. Disponible en: https://bit.ly/3H6bewm

5. Research Triangle Park International (RTI International). Caso de inversión a favor del control del tabaco en El Salvador. San Salvador. Fondo Solidario para la SaludMinisterio de Salud. 2019. 15 p. Disponible: https://bit.ly/3tWdQZY

6. Organización Mundial de la Salud. Convenio Marco de la OMS para el Control del Tabaco. Ginebra. Organización Mundial de la Salud. 2005. 42 p. Disponible: https://apps.who.int/iris/bitstream/ handle/10665/42813/9243591010.pdf

7. Organización Panamericana de la Salud. Informe sobre el control del tabaco en la Región de las Américas. Washington, DC.OPS-OMS. 2018.73 p. Disponible: https:// iris.paho.org/handle/10665.2/49237

8. Vázquez A, Sesma S, Hernández M. El consumo de tabaco en los hogares en México: resultados de la Encuesta de Ingresos y Gastos de los Hogares, 19842000. Rev. Salud Pública Mex. 2002;44(1):7681. Disponible: http://www.scielo.org. $\underline{\mathrm{mx} / \text { scielo.php?script=sci arttext\&pid }}$ $=$ S0036363420020000700012

9. Moreno Altamirano L. Epidemiología Clínica. 3.a edición. México. McGRAWHILL; 2013. 309P.

10. Argimon J, Jiménez J. Métodos de investigación clínica y epidemiológica. 4.a edición. Barcelona. ELSEVIER. 2013. 399 p.

11. Ministerio de Salud. Sistema de Morbi Mortalidad en la Web. Ministerio de Salud. El Salvador. 2019. Fecha de consulta: 20 junio 2020. Disponible en: https://simmow.salud. gob.sv/

12. Ministerio de Economía. Encuesta de Hogares de Propósitos Múltiples 2019. San Salvador. Dirección General de Estadísticas y Censos (Digestyc). 2020. 35p. Disponible en: http://www.digestyc.gob.sv/index.php/ novedades/avisos/965-ya-seencuentradisponible-la-encuesta-de-hogares-depropositos-multiples-2019.pdf

13. Restrepo L, González J. De Pearson a Spearman. Rev Colom Cienc Pecua 2007;20(2):0120-0690. Disponible: https:// www. redalyc.org/pdf/2950/295023034010. $\underline{\mathrm{pdf}}$ 
14. Reynales-Shigematsu L. El control del tabaco, estrategia esencial para reducir las enfermedades crónicas no transmisibles. Rev Salud Publica Mex. 2012;54:323331. Disponible en: https://www. medigraphic.com/cgibin/new/resumen. cgi?IDARTICULO=34341

15. Fernández E, Figueroa D. Tabaquismo y su relación con las enfermedades cardiovasculares. Rev Haban Cienc Méd. 2018;17(2):225-235. Disponible en: http:// www.revhabanera.sld.cu/index.php/rhab/ article/view/2044/2043

16. Fernández E, Figueroa D. Tabaquismo y su relación con las enfermedades cardiovasculares. Rev Haban Cienc Méd. 2018;17(2):225-235. Disponible: http:// scielo.sld.cu/scielo.php?script=sci arttext\&pid=S1729-519X2018000200008

17. Organización Panamericana de la Salud (OPS). Las ENT de un vistazo: mortalidad por enfermedades no transmisibles $y$ prevalencia de sus factores de riesgo en la Región de las Américas. Washington, D.C. OPS. 2019. 31 p. Disponible: https://iris.paho. org/handle/10665.2/51752

18. Convenio Marco de la Organización Mundial de la Salud para el Control de Tabaco en El Salvador. Informe de la evaluación conjunta de Necesidades. 2016. 5 p. Disponible en: https://www.who.int/fctc/implementation/ needs/Informe-Evaluacion-NecesidadesCMCT-el-salvador-2016.pdf

19. Bolet M, Suárez M. Algunas reflexiones sobre el tabaquismo como factor de riesgo para diferentes enfermedades. Rev cubana Med Gen. 2003;19(4). Disponible en: http:// scielo.sld.cu/scielo.php?script=sci arttext\& pid $=$ S0864-21252003000400001
20. Suárez N, Campos E. Prevalencia y percepción del riesgo del tabaquismo en el área de salud del policlínico "Dr. Jorge Ruiz Ramírez". Rev Cubana Salud Pública. 2010;36(2):864-3466. Disponible: http:// scielo.sld.cu/scielo.php?script=sci arttext\& pid=S0864-34662010000200005

21. Reynales-Shigematsu LM. El control del tabaco, estrategia esencial para reducir las enfermedades crónicas no transmisibles. Rev Salud Publica Mex. 2012;54(3):323331. Disponible en: https://www. medigraphic.com/cgibin/new/resumen. cgi?!DARTICULO=34341

22. Ley para el Control del Tabaco. El Salvador. 28 de julio 2011. Disponible en: http://asp. salud.gob.sv/regulacion/pdf/reglamento/ reglamento ley control del tab aco.pdf

23. Drope J, Schluger N, Cahn Z, Drope J, Hamill S, Islami F, et al. The Tobacco Atlas. Atlanta. Sociedad Americana del CÁNCER. 2018. 52p. Disponible en: https://files.tobaccoatlas. org/wpcontent/uploads/2018/03/ TobaccoAtlas 6thEdition LoRes.pdf

24. Castañeda O, Segura O, Parra A. Prevalencia de enfermedades crónicas no transmisibles, Trinidad-Casanare. Rev.médica Risaralda. 2018;24(1). Disponible: http://www.scielo. org.co/scielo.php?script=sci arttext\&pid $=$ S0122-06672018000100007

25. Ministerio de Salud. Cuarta encuesta nacional sobre consumo de sustancias psicoactivas en población escolar de El Salvador, 2018. San Salvador. Instituto Nacional de Salud. 2019. 104 p. Disponible en: http://ins.salud.gob. sv/wp-content/ uploads/2019/05/Cuarta- encuesta-sobreconsumo-drogas.pdf 